\title{
Neusiedler See
}

In unserer schnelllebigen, von ökonomischem Druck geprägten Zeit ist es oftmals schwierig, der Natur die Ruhe und den Raum zu geben, den sie benötigt. Der Ausgleich zwischen der Nutzung unserer Umwelt durch den und dem Schutz vor dem Menschen wird zu einer immer neuen Herausforderung, aber auch Verpflichtung gegenüber nachfolgenden Generationen.

Der Neusiedler See und der Seewinkel sind ein gutes Beispiel für eine Region, in der man sich dieser Herausforderung seit langem stellt, und - man darf sagen - zumindest teilweise auch gemeistert hat. Der See ist heute Teil eines Naturschutzgebiets, im Süden dies- und jenseits der Staatsgrenze auch eines Nationalparks und gehört zum Natura-2000-Netzwerk. Er ist aber auch Ziel für tausende UrlauberInnen, FreizeittouristInnen und WassersportlerInnen aus dem In- und Ausland.

Während in den letzten Jahren zahlreiche Arbeiten zum naturschutzfachlichen Wert des Sees und der Region erschienen sind, fehlt eine aktuelle zusammenfassende Darstellung aus wasserwirtschaftlicher Sicht. Diese Lücke soll mit dem vorliegenden Themenheft geschlossen werden.

Im Eingangsartikel bieten Sailer und Maracek einen Überblick über die wasserwirtschaftlichen Grundlagen des Neusiedler Sees. Besonderes Augenmerk wird dabei auf die Wasserbilanz und die Regelung des Wasserstands gelegt. Die Autoren beschreiben in ihrer Arbeit die Langzeitveränderungen des Wasserstands im Laufe des 20. Jahrhunderts, beginnend mit dem Bau eines Kanals zwischen dem Neusiedler See und der Raab Ende des 19. Jahrhunderts bis hin zur neuen Wehrbetriebsordnung 2011. In Anbetracht der Tatsache, dass der See wesentlich von Niederschlag und Verdunstung geprägt ist, werden als zentrale Herausforderungen für die Zukunft die globale Erwärmung, die Gefahr einer Verlandung und das Schilfmanagement diskutiert.

Csaplovics widmet sich in seinem Artikel dem Schilfgürtel des Neusiedler Sees. Dieser ist seit dem 19. Jahrhundert einerseits durch unterschiedliche Nutzungsansprüche (Landwirtschaft, Schilfwirtschaft, Fischerei/Jagd, Tourismus), andererseits durch Schutzbestrebungen geprägt. Csaplovics zeichnet die spannende Geschichte des Schilfgürtels von der Antike bis in die Neuzeit nach und weist auf die Gefahren für das, nach dem Donau-Delta zweitgrößte zusammenhängende Schilfgebiet Europas hin. Dazu gehören eine zunehmende Degradation der Schilfbestände und eine Ausweitung der offenen Wasserflächen innerhalb des Schilfgürtels. Als Voraussetzung für allfällige Managementmaßnahmen werden unter anderem eine periodische, grenzüberschreitend harmonisierte Inventur und Analysen der Schilfstruktur und -klassifikation hervorgehoben.

Die Bedeutung des Schilfgürtels für die Wasserqualität des Sees ist seit langem bekannt und wurde durch Analysen zur Nährstoffbilanz des Sees eindrucksvoll unterstrichen. Die Eintragspfade, Umsetzungsprozesse und Langzeitveränderungen der Nährstoffe werden in diesem Themenheft von Wolfram et al. behandelt. Ausgehend von älteren Untersuchungen vom Anfang der 1980er-Jahre und unter Einbeziehung neuerer Erkenntnisse aus einem laufenden EU-INTERREG-Projekt (REBEN) werden die Nährstofffrachten der Wulka, des größten Zubringers zum See, sowie Veränderungen im Chemismus des Wulkawassers beim Durchtritt durch den Schilfgürtel analysiert. Die Erkenntnisse zu den räumlichzeitlich äußerst dynamischen Transport- und Umsetzungsprozessen von Nährstoffen, in Verbindung mit hydraulischen Modellen zum Austausch zwischen offenem See und Schilfgürtel, sind eine wichtige Grundlage für die Erarbeitung eines wasserwirtschaftlichen Managementplans für den Neusiedler See.

Im Gegensatz zum Kenntnisstand über die Nährstoffdynamik ist die Belastung des Neusiedler Sees mit anthropogenen Spurenstoffen deutlich weniger untersucht. Diesem Thema widmen sich Zessner et al. Der Artikel fasst den aktuellen Wissensstand zur Belastung des Sees mit nationalen oder gemeinschaftlich geregelten anthropogenen Spurenstoffen zusammen. Darüber hinaus behandeln die 


\section{Editorial}

AutorInnen die Eintragspfade sowie das mögliche Umweltverhalten von Spurenstoffen in den bzw. im See. Die Einhaltung der Umweltqualitätsnormen gemäß EU-Wasserrahmenrichtlinie ist für die meisten Stoffe gegeben, für einzelne Stoffe ist hingegen eine Zielverfehlung anzunehmen. Trotz einer begrenzten Datenlage und Unsicherheiten in der Bewertung zeigen die Autorlnnen eine ganz besondere Vulnerabilität des Sees für die Belastung mit anthropogenen Spurenstoffen auf, die in einer zukünftigen Managementstrategie für den See zu berücksichtigen ist.

Den Schlusspunkt dieses Themenheftes bildet ein weiterer Aspekt zur Wasserqualität des Neusiedler Sees, nämlich die mikrobiologisch-hygienische Belastung. Herzig et al. stellen einen historischen Abriss an den Beginn ihrer Arbeit. Auf Grundlage einer statistischen Analyse von Langzeitdaten und deren Verknüpfung mit Wasserqualitätsparametern und Wetterdaten werden EU-Badestellen mit ausgezeichneter Wasserqualität, aber auch Hotspots fäkaler Belastung identifiziert. Erkenntnisse aus einer Pilotstudie für ein nachhaltiges Gesamtkonzept zum Management des mikrobiologisch-hygienischen Zustandes des Neusiedler Sees münden abschließend in Vorschläge für ein künftiges Monitoring fäkaler Verschmutzung.

Mit fünf Beiträgen wird in diesem Themenheft der Status quo zur Hydrologie und Wasserwirtschaft, zum Schilfgürtel und zur stofflichen Belastungssituation (Nährstoffe, anthropogene Spurenstoffe, fäkale Verschmutzung) des Neusiedler Sees beschrieben. Auf Basis langjähriger Erfahrung der Autorlnnen und unter Einbeziehung von Erkenntnissen aus neueren Studien wird die bisherige Entwicklung nachgezeichnet und durch Aufzeigen von Kausalzusammenhängen eine Grundlage zur Abschätzung der künftigen Entwicklung geschaffen. Dabei bestehen jedoch nach wie vor Wissenslücken, welche durch ein intensiviertes, langfristiges Monitoring und die Erforschung spezifischer Fragestellungen zu schließen sind - eine Erkenntnis, die allen Beiträgen in diesem Themenheft gemeinsam ist. Schließlich gewährleistet nur fundiertes Wissen in interdisziplinärer Abstimmung, effiziente Managementmaßnahmen zu formulieren, um langfristig den Schutz- wie auch den Nutzungsansprüchen an den Neusiedler See gerecht zu werden.

\section{Mag. Dr. G. Wolfram ( $\bowtie)$}

DWS Hydro-Ökologie $\mathrm{GmbH}$,

Zentagasse 47,

1050 Wien, Österreich

georg.wolfram@dws-hydro-oekolo-

gie.at

\section{ao.Univ.-Prof. DI Dr. M. Zessner}

Institut für Wassergüte und

Ressourcenmanagement, Technische

Universität Wien,

Karlsplatz 13,

1040 Wien, Österreich

mzessner@iwag.tuwien.ac.at 